\title{
Tsafon
}

Revue d'études juives du Nord

$81 \mid 2021$

Des synagogues à travers les âges Lieux de prières, lieux d'études et autres fonctions

\section{La synagogue de Lille et les deux guerres mondiales}

\section{Rudy Rigaut}

\section{(2) OpenEdition}

\section{Journals}

Édition électronique

URL : https://journals.openedition.org/tsafon/3810

DOI : 10.4000/tsafon.3810

ISSN : 2609-6420

Éditeur

Association Jean-Marie Delmaire

Édition imprimée

Date de publication : 1 juillet 2021

Pagination : 89-108

ISSN : 1149-6630

\section{Référence électronique}

Rudy Rigaut, «La synagogue de Lille et les deux guerres mondiales », Tsafon [En ligne], 81 | 2021, mis en ligne le 01 juillet 2021, consulté le 15 septembre 2021. URL : http://journals.openedition.org/ tsafon/3810; DOI : https://doi.org/10.4000/tsafon.3810 


\section{La synagogue de Lille et les deux guerres mondiales}

Rudy Rigaut*

Au lieu de la noire masure de la rue des Prisons qui avait l'air d'un débris de Ghetto, les israélites Lillois possèdent (désormais) une synagogue monumentale située dans un quartier neuf. ${ }^{1}$

C'est en ces termes que débute l'article de la revue Archives israélites relatant la cérémonie d'inauguration de la synagogue de Lille ayant eu lieu le vendredi 25 septembre $1891^{2}$. Devenu siège consistorial en 1872, Lille se dote ainsi d'un édifice religieux monumental, de style romano-byzantin ${ }^{3}$, digne de son statut.

Construite durant l'été $1890^{4}$, la synagogue de la rue Gauthier de Châtillon (devenue rue Angellier) représente alors le centre de la vie juive dans la ville et le symbole du dynamisme de la communauté religieuse mais son passé témoigne aussi des souffrances subies pendant les périodes troublées de la fin du $\mathrm{XIX}^{\mathrm{e}}$ siècle et surtout de la première moitié du $\mathrm{XX}^{\mathrm{e}}$ siècle marquée par les deux conflits mondiaux.

\footnotetext{
* Docteur en histoire juive contemporaine (Artois-CREHS ; ENS-IHMC), enseignant, correspondant régional du Mémorial de la Shoah et chargé de cours sur le judaïsme à l'université catholique de Lille.

${ }^{1}$ Archives israélites, $\mathrm{n}^{\circ} 40,1^{\mathrm{er}}$ octobre 1891, p. 319.

${ }^{2}$ Sur l'histoire de la synagogue de Lille, voir Danielle Delmaire, Les communautés juives septentrionales 1791-1939. Naissance, croissance, épanouissement, doctorat d'État, Université de Lille-III, 1998, p. 682-705 ; Danielle Delmaire, La synagogue de Lille, 130 ans du judaïsme lillois, Édition Guy Bensoussan, 2019, 108 p ; Rémy Joly, «Petite histoire de la synagogue de Lille », Tsafon, revue d'études juives du Nord, $\mathrm{n}^{\circ} 6-7$, automne 1991, p. 33-41.

${ }^{3}$ Sur l'art architectural des synagogues françaises, voir Dominique Jarrassé, Une histoire des synagogues françaises: entre Occident et Orient, Arles, Actes Sud, coll. « Hebraïca », 1997.

${ }^{4}$ Danielle Delmaire, Les communautés juives de la France septentrionale au XIX ${ }^{e}$ siècle (1791-1914), l'entrée dans la nation, Paris, L'Harmattan, 2008, p. 157.
} 


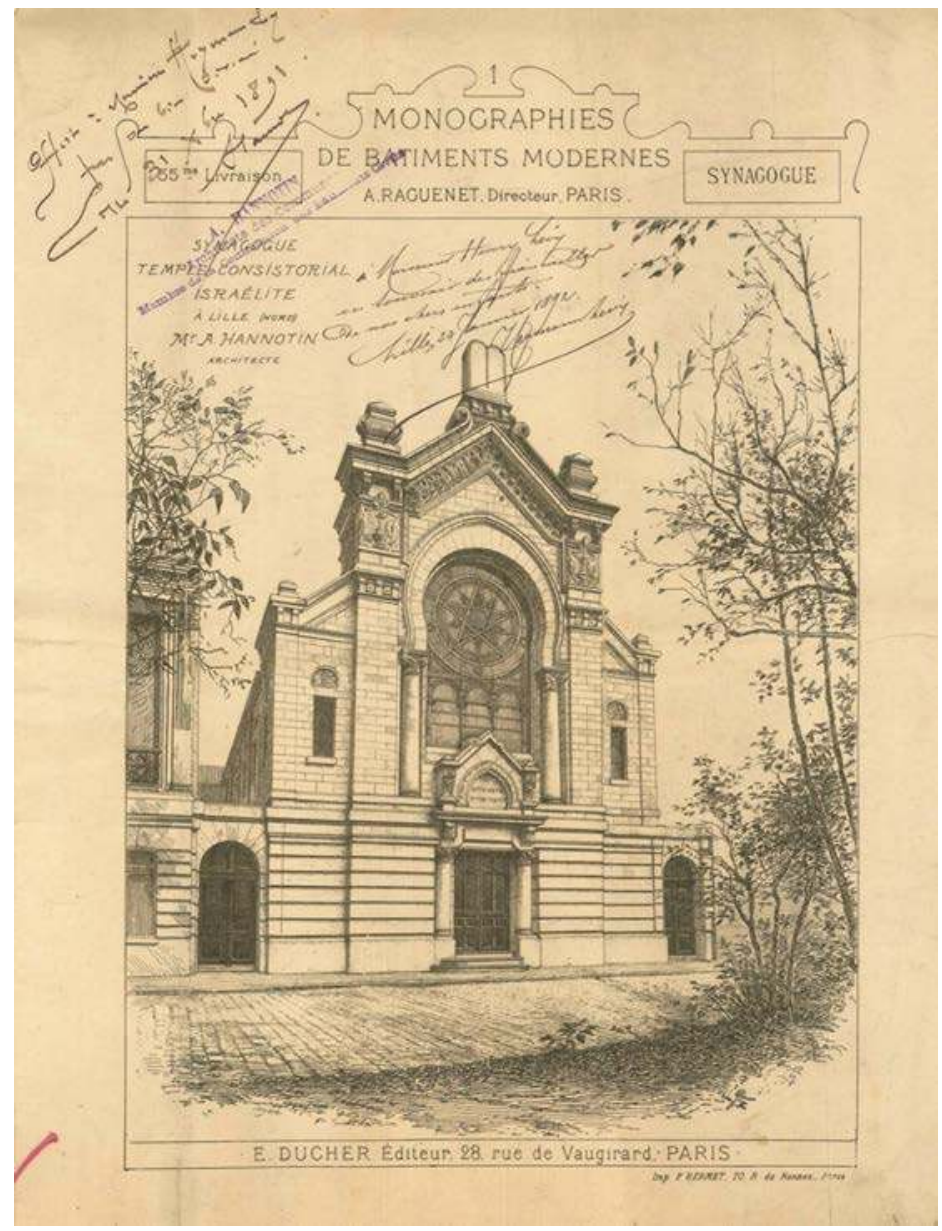

Livret fourni par l'architecte Hannotin

au responsable des travaux de la communauté juive de Lille en $1891^{5}$

Source : communauté juive de Lille

Il ne s'agit pas seulement de retracer à grands traits l'histoire de la synagogue de Lille durant les deux guerres mondiales et d'analyser les liens établis entre ces deux événements majeurs et ce lieu de culte, devenu également lieu de mémoire ou de souvenir ${ }^{6}$ et de commémorations, mais

\footnotetext{
${ }^{5}$ On peut observer que la rosace de la façade était alors une étoile à dix branches et non à six comme cela est le cas aujourd'hui.

${ }^{6}$ L'expression « lieux de mémoire » a été forgée et popularisée par Pierre Nora (sous la dir.), Les Lieux de mémoire, t. 1, Paris, éditions Quarto Gallimard, 1997, p. 15-21. Les historiens Serge Barcellini et Annette Wieviorka lui préfèrent « lieux du souvenir » car « une partie des plaques, des stèles ou des monuments, surtout ceux érigés dès l'aprèsguerre, l'ont été pour que les Hommes se souviennent d'abord de ceux qui avaient perdu la vie. Ce sont donc des lieux du souvenir, et non des lieux de mémoire dans le sens défini par Pierre Nora, et repris dans l'édition 1993 du Grand Robert de la Langue française : 'Unité significative, d'ordre matériel ou idéel, dont la volonté des hommes ou le travail
} 
l'étude vise aussi à appréhender autrement la manière dont la communauté, au sens cultuel ou associatif du terme, a traversé les deux conflits et les initiatives prises, après les guerres, pour inscrire les pratiques commémoratives dans un bâtiment qui a comme vocation première la pratique religieuse.

\section{La synagogue de Lille et la Grande Guerre}

Marqué par l'occupation de la ville par les troupes allemandes d'octobre 1914 à octobre 1918 mais aussi par la répression sévère et les restrictions multiples qui en découlent, le contexte de la Première Guerre mondiale a inévitablement perturbé les offices religieux organisés à la synagogue.

Le grand rabbin de la communauté lilloise se trouve mobilisé pendant la durée de la guerre. En poste depuis 1907, Edgar Sèches est ainsi nommé aumônier de la place militaire de Lille, mais, à la suite de la mobilisation générale et de l'occupation de la ville, il devient aumônier du $21^{\mathrm{e}}$ corps d'armée, puis il est affecté, en novembre 1914, à la place d'Épinal et, début 1915, à celle de Lyon où il devient grand rabbin intérimaire en $1916^{7}$.

La situation de la synagogue permet d'appréhender l'impact de la guerre et de l'occupation allemande sur la vie juive locale. Malgré les départs précipités de deux de ses cadres : celui de son grand rabbin Sèches et de son président Alfred Bernheim, en fonction de 1904 à 1914, date à laquelle il se fixe définitivement à Paris $^{8}$, le maintien des effectifs communautaires durant les années de guerre ${ }^{9}$ permet d'y organiser des cérémonies religieuses. Ayant conservé des liens avec les membres malgré son éloignement, Edgar Sèches adresse en septembre 1915 une lettre à L'Univers israélite fournissant des renseignements précieux car rares sur la situation lilloise à cette date. Surtout, il souligne la dimension politique prise par le lieu et les activités qui s'y déroulent :

\footnotetext{
du temps a fait un élément symbolique'». Serge Barcellini et Annette Wieviorka, Passant, souviens-toi! Les lieux du souvenir de la Seconde Guerre mondiale en France, Paris, Plon, 1996, p. 7.

${ }^{7} L^{\prime}$ Univers israélite, $\mathrm{n}^{\circ} 45,14$ juillet 1916, p. 460. Voir aussi sa notice biographique dans Jean-Philippe Chaumont et Monique Lévy (sous la dir.), Dictionnaire biographique des rabbins et autres ministres du culte israélite. France et Algérie, du Grand Sanhédrin (1807) à la loi de Séparation (1905), Paris, Berg international, p. 656-658.

${ }^{8} L^{\prime}$ Univers israélite, $\mathrm{n}^{\circ} 10,13$ novembre 1936, p. 156.

${ }^{9} L^{\prime}$ 'Univers israélite, ${ }^{\circ}$ 20, 24 janvier 1919, p. 471-472.
} 
Dans la synagogue de Lille comme dans celle de Bruxelles, le ministre officiant continue à réciter tous les samedis la prière pour la République à la barbe des Allemands. ${ }^{10}$

Selon le grand rabbin, y tenir un office représente en temps ordinaire un devoir religieux mais constitue aussi, dans ce contexte troublé, un acte de résistance dont la synagogue devient le lieu symbolique. Malgré les arrestations, les dégâts matériels et les départs des deux principaux responsables communautaires, des cérémonies religieuses continuent à y être célébrées par le ministre officiant Anselme Lévy.

Pourtant, le lieu de prières n'a pas été épargné par les aléas de la guerre. Survenue les 10-11 janvier 1916, l'explosion de la poudrière des « dix-huit ponts ${ }^{11}$ a partiellement atteint la synagogue qui se trouve à proximité du quartier touché. Fragilisée, la toiture finit même par s'écrouler en janvier 1921. Pour financer les travaux dont le montant est estimé à environ 35000 francs, Alexandre Heymann, président de la commission administrative de l'association cultuelle, lance une campagne de souscription qui permet de réunir près de la moitié de la somme. Parallèlement, il adresse plusieurs lettres à la municipalité qui, en tant que propriétaire du lieu, finit par accepter de débloquer des fonds pour couvrir une partie des dépenses. Approuvée par le conseil municipal lors de sa séance du 12 mai 1921, la réparation de la toiture s'achève en août $1922^{12}$.

Après cette date, le lieu de prières doit encore faire l'objet d'importants travaux de peinture, d'électricité et de chauffage mais le manque d'entretien pendant la guerre ainsi que les dégâts causés à la toiture et la rosace pèsent durablement sur la vie communautaire ${ }^{13}$. Le procès-verbal de la séance de la commission administrative du 26 décembre 1923 indique aussi que les rouleaux de la Torah ont été

\footnotetext{
${ }^{10}$ L'Univers israélite, $\mathrm{n}^{\circ} 2,17$ septembre 1915, p. 48.

${ }^{11}$ Dépôt de munitions aménagé par l'armée allemande dans le quartier de Moulins. L'explosion anéantit le quartier et cause la mort de 104 civils et 30 soldats allemands. Sur ce sujet, lire notamment Alain Cadet, L'explosion des Dix-huit Ponts. Un «AZF » lillois en janvier 1916, Lille, Les Lumières de Lille, 2015.

${ }^{12}$ Archives municipales de Lille (AML) : 2 M 174. Il s'agit d'un dossier contenant de nombreux documents relatifs aux travaux de réfection de la synagogue : procès-verbaux d'adjudication et de réception définitive, délibérations, rapports, plan de la rosace, cahier des charges, devis, notes, correspondance.

${ }^{13} \mathrm{Ibid}$. À titre d'exemple, le coût de la réfection de la rosace représentant encore à l'époque une étoile à dix branches s'élève à 4100 francs. En 1927, la somme de 1000 francs a été offerte à la ville par l'association cultuelle pour couvrir une partie des frais, et ce malgré des recettes modestes. Voir aussi Danielle Delmaire, Les communautés juives septentrionales..., op. cit., p. 702-704.
} 
endommagés lors des combats. Le grand rabbin Salomon Poliakof, nommé en 1921 à la tête d'une communauté alors éprouvée par l'occupation allemande et les violences, se charge notamment des démarches visant à la réparation des rouleaux ${ }^{14}$. En 1930, les problèmes matériels ne sont toujours pas résolus. Le $1^{\text {er }}$ octobre, une partie du plafond située au-dessus de la galerie des dames s'est même effondrée lors de l'office religieux. Aucune victime n'a été à déplorer. En revanche, quelques semaines plus tard, la vétusté du plancher d'une des salles du rez-de-chaussée a engendré la chute d'un enfant ${ }^{15}$.

Après la guerre, la synagogue se charge de ce passé et devient, en sus de sa fonction de lieu de prières, un lieu de mémoire ou de souvenir. Ce choix n'est pas anodin. En effet, la synagogue fournit un cadre assurant l'intimité, la discrétion du recueillement et garantit ainsi le développement d'une partie de l'activité commémorative dans un entre-soi ancré dans un lieu clairement identifié et accessible. Afin d'en faire un lieu du souvenir et un point de fixation possible pour des rassemblements commémoratifs, une plaque est apposée sur l'un des murs, venant rappeler la mort au combat de 14 membres de la communauté ${ }^{16}$. Elle porte les mots suivants : « À la mémoire des enfants de la communauté israélite de Lille morts pour la France » suivis des noms des 14 individus classés, à une exception près ${ }^{17}$, dans l'ordre alphabétique. Avec ce bilan, la communauté de Lille est, de toutes les associations cultuelles israélites du Nord et du Pas-deCalais, la plus durement touchée par les pertes humaines lors de la Grande Guerre ${ }^{18}$.

En raison de l'engagement de la communauté pendant la Grande Guerre mais aussi de la renommée dont jouit localement son grand rabbin, la synagogue finit même par s'imposer comme un site commémoratif majeur dans la deuxième moitié des années $1930^{19}$. Ainsi, lors du $18^{\text {ème }}$

14 Cahier des PV de la commission, cité par Danielle Delmaire, Les communautés juives septentrionales..., op. cit., p. 756.

${ }^{15}$ AML : 2 M 174.

${ }^{16}$ La date d'inauguration de la plaque n'a pas été retrouvée.

${ }^{17}$ N'étant pas aligné dans la liste, le nom de Léon Bloch a, selon toutes vraisemblances, été ajouté après coup. Voir la reproduction photographique en fin d'article.

${ }^{18}$ Voir Rudy Rigaut, « Les communautés juives du Nord et du Pas-de-Calais et la Grande Guerre », dans Xavier Boniface et Jean Heuclin, Diocèses en guerre 1914-1918, L'Église déchirée entre Gott mit uns et le Dieu des armées, Villeneuve d'Ascq, Presses universitaires du Septentrion, 2018, p. 83-100.

${ }^{19}$ Sur la construction de lieux du souvenir et l'organisation de cérémonies impliquant des groupes juifs dans l'entre-deux-guerres, voir Philippe Landau, «Le monument de Douaumont (1938). L'appel à la fraternité dans une France tourmentée », Archives juives. Revue d'histoire des Juifs de France, 28/1, 1995, p. 86-90 ; Dominique Jarrassé, « Le 
anniversaire de l'armistice, une cérémonie publique se déroule à la synagogue en présence des autorités civiles, de délégations d'anciens combattants français et alliés, du président de la communauté Alex Heymann et du grand rabbin de Lille Léon Berman, lui-même ancien combattant et ancien aumônier militaire. Inquiet face à la poussée antisémite en France, le rabbin saisit l'occasion pour mettre en garde l'assistance :

$[\ldots]$ contre les barrières qui semblent se dresser devant les différentes classes et familles spirituelles du pays [...] En vérité, les divergences d'opinion et de point de vue entre les enfants d'une même patrie n'ont pas plus d'importance qu'un cheveu. $^{20}$

Dans cet extrait de son discours prononcé en 1936, le grand rabbin de Lille exprime l'espoir que le concours patriotique et les sacrifices consentis par les Juifs ${ }^{21}$ lors de la Grande Guerre ont permis de ne plus mettre en cause leur appartenance nationale. Les commémorations de la Première Guerre mondiale organisées à la synagogue à cette époque mettent en scène l'attachement à la France mais se font aussi l'écho des inquiétudes et des questionnements qui surgissent dans les années 1930.

\section{Un symbole et un lieu de mémoire des persécutions antisémites de la Seconde Guerre mondiale}

Le déclenchement de la Seconde Guerre mondiale bouleverse les activités synagogales et l'équilibre précaire retrouvé de la communauté de Lille. En poste depuis le milieu des années 1930, son grand rabbin Léon Berman, devenu aumônier militaire en 1939, renonce à revenir dans le secteur après la défaite militaire et l'occupation de la ville par les troupes allemandes ${ }^{22}$.

Les politiques et les violences antisémites qui s'accumulent pendant la période n'épargnent pas la synagogue lilloise qui devient l'incarnation architecturale des persécutions locales subies. En effet, on trouve trace,

rêve brisé d'union sacrée. Les monuments israélites de la Grande Guerre », Archives juives. Revue d'histoire des Juifs de France, 33/1, 2000, p. 82-92.

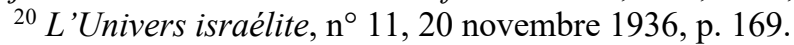

${ }^{21} \mathrm{~L}$ 'appartenance juive ne se limitant pas à sa dimension religieuse, une majuscule est donnée au substantif « Juif » dans cet article.

${ }^{22}$ Domicilié à Cannes, Léon Berman est finalement arrêté avec sa famille puis déporté à Auschwitz par le convoi $n^{\circ} 61$ au départ de Drancy le 28 octobre 1943. CDJC : C61-6. Liste du convoi de déportation $n^{\circ} 61$. 
dans les archives départementales du Nord, de documents faisant mention d'actes de dégradation volontaire visant les parties extérieures du lieu de culte. Ces sources policières sont précieuses pour reconstituer les faits. Elles permettent aussi de décrire les profils des auteurs, leurs motivations et montrent qu'en dépit de la rafle du 11 septembre 1942, où environ 150 personnes ont été arrêtées à Lille pour être déportées vers Auschwitz via le camp de transit de Malines, l'antisémitisme sévit encore sur le territoire et vise désormais les biens.

Le 7 juin 1942 vers 13 heures, trois individus ont été surpris par deux gardiens de la paix de la brigade cycliste en plein acte de vandalisme. Les policiers les ont interpellés alors qu'ils jetaient des pierres en direction des vitres du lieu de prières.

Un rapport de police est établi dès le lendemain des faits. Tapé à la machine par Monsieur Godec, commissaire de police du $\mathrm{V}^{\mathrm{e}}$ arrondissement de la ville, le document est transmis au préfet régional, au procureur et à l'intendant de police ${ }^{23}$. Le rapport fournit des renseignements assez précis sur les auteurs des faits. Pierre Dereume, 17 ans, étudiant, est domicilié rue Solferino ; David Deceuninck, 16 ans, apprenti mouleur demeure rue Nationale; Jacques Blamart, 18 ans, interprète est installé rue de Douai. Outre leur jeune âge, le rapport indique que « les deux premiers sont membres du Parti Franciste; le dernier est affilié au Parti Populaire Français ». Ces mouvements collaborateurs parisiens ont été autorisés par 1'OFK $670^{24}$ à développer leurs activités dans le Nord et le Pas-de-Calais après l'attaque allemande contre l'URSS le 22 juin 1941. Sur le plan sociologique, ces organisations recrutent essentiellement dans l'agglomération lilloise, considérée comme « un haut lieu de la collaboration politique », avec « une forte minorité de jeunes dans une organisation d'adultes $»^{25}$. Par ailleurs, le passage à l'acte s'inscrit dans un contexte de concurrence, durant l'été 1942, entre les mouvements collaborateurs nordistes marqué pour le Parti populaire français (PPF) de Jacques Doriot par «des grandes campagnes d'affichage, des distributions de tracts en pleine rue par des équipes en

\footnotetext{
${ }^{23}$ Archives départementales du Nord (ADN) : 1 W 1090.

${ }^{24}$ Oberfeldkommandantur, basée à Lille, la 670 est chargée de commander et d'administrer le Nord et le Pas-de-Calais.

${ }^{25}$ Marc Sueur, «La collaboration politique dans le département du Nord», Revue d'histoire de la Deuxième Guerre mondiale, 1984, n 134, p. 4-45, ici p. 32-33. Sur la sociologie de ces groupes, voir aussi Laurent Joly, Les collabos : treize portraits d'après les archives des services secrets de Vichy, des RG et de l'Épuration, Paris, Tallandier, 2019, 256 p. [1ère éd. 2011].
} 
uniformes, des destructions de bustes de la République volés dans les bâtiments officiels, des exactions contre les familles et les biens juifs $»^{26}$. Cependant, l'émulation de l'été 1942 n'est que provisoire et l'activité de ces groupuscules décline très rapidement au cours des mois suivants ${ }^{27}$.

Le fonctionnaire de police conclut son rapport en précisant que les trois individus ont fait l'objet d'un procès-verbal pour «dommages volontaires à la propriété d'autrui » mais remis en liberté après l'interrogatoire. Bien que le caractère antisémite ne semble guère faire de doute en raison de l'appartenance politique des auteurs à des mouvements de type fascistes, collaborationnistes et ouvertement antijuifs ${ }^{28}$, ce mobile n'est ni mentionné ni retenu concernant cet acte de dégradation. Cela n'a rien d'étonnant. Le rapport et le procès-verbal reflètent ce contexte idéologique spécifique où l'antisémitisme devient une norme juridique et morale pour une partie de la population mais aussi pour l'ensemble des administrations françaises qui doivent mettre en œuvre les politiques antisémites du gouvernement de Vichy et de l'occupant ${ }^{29}$.

En complément de son rapport, le commissaire de police joint un article publié en page trois du quotidien pro-allemand $L$ 'Écho $d u$ Nord $^{30}$ du 8 juin 1942. Pour l'auteur de cet article intitulé «' 'Mesures' contre les Juifs », le mobile antisémite, dont il se réjouit, est évident :

\begin{abstract}
Si la pratique de la religion juive en principe est interdite, il n'en existe pas moins encore à Lille une synagogue qui, encore maintenant, arbore insolemment sur des vitraux tous les attributs de la «race élue » : rosaces, triangles, étoiles et toute la kyrielle des figures géométriques plus ou moins régulières, chères aux fils de Sion. Or, il y a dans notre ville beaucoup de jeunes gens à qui cela n'eut pas le don de plaire, et qui, plus audacieux que les autres décidèrent d'y mettre bon ordre. Hier, dimanche, ils se mirent donc en devoir de démolir les vitraux à coups de pierre.

Malheureusement, des agents qui passaient par là les interrompirent au beau milieu de leur travail. Conduits au commissariat, procès-verbal leur fut dressé après quatre heures de détention arbitraire.
\end{abstract}

\footnotetext{
${ }^{26}$ Étienne Dejonghe et Yves Le Maner, Le Nord-Pas-de-Calais dans la main allemande 1940-1944, Lille, La Voix du Nord Éditions, 2019, p. 256.

${ }^{27}$ Ibid., p. 257-260.

${ }^{28}$ Tal Bruttmann, « Du militantisme à l'action. L'activisme antisémite des ultras de la Collaboration », Revue d'Histoire de la Shoah, vol. 198, n 1, 2013, p. 179-193.

${ }^{29}$ Marc Olivier Baruch, Servir l'État français. L'administration en France de 1940 à 1944, Paris, Fayard, 1997, 737 p.; Tal Bruttmann, Au bureau des affaires juives. L'administration française et l'application de la législation antisémite (1940-1944), Paris, La Découverte, 2005, $287 \mathrm{p}$.

${ }^{30}$ Sur la presse locale pendant l'Occupation, voir Jean-Paul Visse, La presse du Nord et du Pas-de-Calais au temps de "L'Écho du Nord" : 1819-1944, Villeneuve d'Ascq, Presses universitaires du Septentrion, 2004, 279 p.
} 
Il est pénible de voir que des jeunes gens, dont le seul crime est de ne pas aimer les juifs... et de le montrer soient emmenés au poste tout comme de vulgaires repris de justice ou condamnés de droit commun.

Si la municipalité, ainsi qu'il se doit, avait fait enlever les signes hébraïques de l'endroit où ils s'étalent impudiquement et d'une manière provocatrice, ces jeunes gens ne se seraient pas trouvés dans l'obligation d'accomplir eux-mêmes ce travail.

Mais l'administration et la Police ne sont pas encore, à ce qu'il parait, tout à fait au diapason des idées nouvelles... ${ }^{31}$

Partant des faits, l'auteur de l'article généralise et reprend la rhétorique antisémite propre à ces mouvements politiques; il déplore avant tout le maintien de la synagogue dans la ville, il stigmatise la « race élue » et des allusions sont faites, à peine voilées, aux symboles et au fantasme de la collusion judéo-maçonniques. Enfin, est déplorée l'attitude jugée trop tolérante voire laxiste des autorités à l'égard des «signes hébraïques » et, à l'inverse, jugée trop sévère à l'encontre des vandales, accusant à demi-mot les autorités de s'opposer aux nouvelles tendances idéologiques. Libérant la parole antisémite dans la presse, l'abrogation du décret Marchandeau le 16 août 1940 permet à l'auteur de l'article d'afficher ouvertement un antisémitisme d'idées et d'actions.

Malgré le déclin de ces groupuscules, la synagogue reste pour leurs membres une cible privilégiée d'attaque antisémite. Dans la nuit du 28 au 29 mars 1943, des projectiles ont été de nouveau lancés vers les vitres du lieu de prières par un dénommé Jean Bertrand, également issu du parti franciste. Dans le rapport qu'il adresse au préfet du Nord, le commissaire aux renseignements généraux précise :

Dimanche matin, l'intéressé s'est vanté d'avoir fracturé des vitraux à la synagogue et ajouté que prochainement l'Église protestante de Lille et l'habitation du pasteur Cheradame recevraient une visite semblable. En outre, il a été prévu que la prochaine expédition contre ces lieux religieux aurait pour but l'enlèvement de différentes pièces de mobilier qui seraient destinées à garnir les permanences du parti franciste. ${ }^{32}$

L'effraction et la tentative de cambriolage de la synagogue amènent la mairie, en tant que propriétaire du bâtiment, à réaliser en mai 1943 un inventaire précis du mobilier et à envisager la transformation du lieu en

\footnotetext{
${ }^{31}$ ADN : $1 \mathrm{~W} 1090$.

${ }^{32}$ ADN : $1 \mathrm{~W}$ 2321. Rapport du commissaire aux renseignements généraux au préfet du Nord daté du 30 mars 1943.
} 
garde-meubles pour les sinistrés ou pour les immeubles réquisitionnés ${ }^{33}$. Finalement, le mobilier de la synagogue est épargné et le restera jusqu'à la Libération. Suivie par les services des renseignements généraux, l'enquête sur ce nouvel acte de vandalisme se révèle assez délicate car l'accusé est également mêlé à des détériorations de matériel et à une affaire de vols dans les locaux de la préfecture du Nord où il travaille comme gardien de la paix ${ }^{34}$.

Avec la Libération, la synagogue redevient le lieu central de la vie religieuse qu'elle était avant-guerre. L'association cultuelle se reconstitue mais pour nombre de familles, le retour est une épreuve. Et pas seulement en raison des dégâts matériels. Certes, la restauration de la légalité républicaine le 9 août 1944 provoque l'abrogation des mesures antisémites mais, dans le Nord et le Pas-de-Calais, les années de l'après-guerre restent empreintes de méfiance voire d'hostilités antijuives comme en témoigne le jet d'une brique sur le toit de la synagogue de Lens lors d'un office religieux début $1947^{35}$ ou les croix gammées dessinées et le mot «Jude » inscrit sur la porte d'entrée et la façade de la synagogue de Lille le dimanche 28 novembre $1965^{36}$.

Après 1945, la synagogue de Lille, forte de son expérience mémorielle de la Grande Guerre, endosse une nouvelle fois la fonction de lieu du souvenir. Des plaques sont ainsi apposées dans les années d'aprèsguerre $^{37}$. Tout d'abord, un ensemble de six plaques de marbre blanc vise à honorer la mémoire de 238 personnes «martyrs victimes de la barbarie nazie morts en déportation pour la France ». On peut établir ici un parallèle avec des pratiques observées dans d'autres lieux de prières. Comme à la synagogue de Lens où une immense plaque de marbre datant de la fin des années cinquante - début des années soixante comporte les noms de 462 personnes, est également affirmée ici l'importance accordée à la conservation des noms. De la même manière qu'à Paris ${ }^{38}$ où un monument commémoratif a été inauguré à l'intérieur de la grande synagogue de la rue de la Victoire le 27 février 1949, on remarque que le lieu de prières

\footnotetext{
${ }^{33}$ AML : 2 M 175.

${ }^{34}$ ADN : 1 W 2321.

${ }^{35}$ Archives Départementales du Pas-de-Calais (ADPC) : 2170 W 215.

${ }^{36}$ Bulletin des Communautés (1965) cité par Danielle Delmaire, La synagogue de Lille, 130 ans du judaïsme lillois, op. cit., p. 97.

${ }^{37}$ Faute de sources communautaires ou de témoignages relatifs à cette période, la date exacte de l'apposition de ces plaques n'a pas été retrouvée. Voir la reproduction photographique en fin d'article.

${ }^{38}$ Simon Perego, Pleurons-les. Les Juifs de Paris et la commémoration de la Shoah (1944-1967), Champ-Vallon, «Époques », 392 p.
} 
peut représenter un cadre privilégié pour développer l'activité commémorative visant, dès l'après-guerre, à mettre en évidence la singularité du sort des Juifs. Dans cet article, nous n'aborderons pas en profondeur les questions relatives aux frontières du groupe fixées par les acteurs du projet. Néanmoins, la plaque appelle plusieurs remarques. Tout d'abord, on peut noter un décalage entre la volonté affirmée dans le texte d'entretenir le souvenir des victimes de la déportation et l'inscription des noms de neuf individus accompagnés de la mention « fusillé » écrite entre parenthèses. Outre l'occultation de l'enjeu de la déportation des Juifs, en l'occurrence l'assassinat, la formule «morts [...] pour la France» témoigne surtout de l'adoption par les groupes juifs locaux de la conception globalisante de la déportation, conduisant à atténuer le caractère spécifique de la Shoah ${ }^{39}$. Elle atteste également de l'influence des cérémonies patriotiques sur les pratiques commémoratives juives, « signe évident d'une acculturation de la mémoire à la France ${ }^{40}$ dans l'immédiat après-guerre selon Annette Wieviorka.

Confirmant cette tendance, la synagogue de Lille abrite une autre plaque plus modeste qui a été apposée « à la gloire des engagés volontaires et anciens combattants juifs de Lille morts au champ d'honneur 19391945 ». Placée à proximité de celle commémorant la mort des soldats de la guerre 1914-1918, elle comporte les noms de 16 individus classés dans l'ordre alphabétique sauf pour trois d'entre eux ayant été ajoutés après coup $^{41}$.

Si la particularité de l'expérience juive est un fait reconnu par beaucoup, ce choix réaffirme le désir de réintégration dans la société française, la tentation de l'universalisation, le poids de la condition victimaire et la volonté de rendre hommage aux combattants afin de mettre également en exergue l'engagement des Juifs ${ }^{42}$. À Lens, la mémoire combattante est également honorée avec la création en mars 1946 d'une section locale de l'union des engagés volontaires anciens combattants juifs (UEVACJ). En revanche, la première commémoration de la rafle est organisée dans le bassin lensois dès septembre 1945, elle ne se déroule pas

\footnotetext{
${ }^{39}$ Sur la conception globalisante de la déportation dans l'immédiat après-guerre, voir Annette Wieviorka, Déportation et génocide. Entre la mémoire et l'oubli, Paris, Plon, $1992,506 \mathrm{p}$.

${ }^{40}$ Annette Wieviorka, «1992. Réflexions sur une commémoration », Annales, ESC, n 3 , mai-juin 1993, p. 709.

${ }^{41}$ Voir la reproduction photographique en fin d'article.

${ }^{42}$ Sur cette tendance à Paris, voir Simon Perego, Pleurons-les. Les Juifs de Paris et la commémoration de la Shoah (1944-1967), op. cit., p. 169-195.
} 
à la synagogue de la ville mais dans la salle Gabilly où se rassemblent 120 personnes $^{43}$.

Organisées à la synagogue de Lille au moins depuis la présidence de Jacques Malamet-Beer au début des années $1980^{44}$, avant Pessah ou à l'occasion de la «journée nationale du souvenir de la déportation » le dernier dimanche d'avril, les commémorations dédiées à la révolte du ghetto de Varsovie, l'un des principaux temps forts commémoratifs de la communauté de Lille, confirment cette volonté d'honorer les victimes mais aussi de glorifier les faits d'armes ${ }^{45}$.

Entre destruction et réparation, intégration et persécutions, histoire et mémoire, la synagogue de Lille offre un étonnant condensé, mis en lumière par des plaques, des cérémonies, des offices, du passé très dense, des épreuves et des souffrances de la communauté juive de Lille dans la première moitié du $\mathrm{XX}^{\mathrm{e}}$ siècle. Cependant, l'histoire de ce lieu de prières et de son association cultuelle depuis 1945 ne doit pas être réduite à la seule transmission de la mémoire des persécutions antisémites pendant la Seconde Guerre mondiale. La variété des activités cultuelles et culturelles proposées, l'aménagement du centre communautaire ${ }^{46}$ et des annexes (salle de réception et de conférences, bibliothèque, logement) au début des années 1980, l'inscription de la synagogue à l'inventaire des monuments historiques en 1984 , la célébration de son centenaire en $1991^{47}$, la réfection

\footnotetext{
${ }^{43}$ Nicolas Mariot et Claire Zalc, Face à la persécution, 991 Juifs dans la guerre, Paris, Odile Jacob, 2010, p. 228-229.

${ }^{44}$ Informations aimablement communiquées par Charles Sulman, président de la commission culturelle de l'association cultuelle israélite (ACI) de Lille (1983-1992), président régional du CRIF et du consistoire depuis 1986, vice-président de l'ACI depuis 1993 puis président d'honneur. (Message électronique, 25 décembre 2018).

${ }^{45}$ Soulignons que depuis le début des années 2000, des commémorations publiques auxquelles participe officiellement la communauté de Lille sont régulièrement organisées à la gare de la ville pour honorer la mémoire des victimes de la rafle du 11 septembre 1942 mais aussi celle des cheminots ayant aidé des Juifs à se sauver. Sur la rafle, voir Danielle Delmaire, «La rafle de Roch hachana, 11 septembre 1942 », Tsafon, revue d'études juives du Nord, $\mathrm{n}^{\circ}$ 9-10, été-automne 1992, p. 15-38; Monique Heddebaut, « Sans arme face à la rafle du 11 septembre 1942 », Tsafon, revue d'études juives du Nord, $\mathrm{n}^{\circ} 70$, automne 2015-hiver 2016, p. 119-168.

${ }^{46}$ Une plaque a été apposée à l'occasion de son inauguration. On peut y lire le texte suivant : «Le 27 novembre 1983 - 21 kislev 5744, ce centre communautaire a été inaugurée par Monsieur Pierre Mauroy Premier Ministre Maire de Lille, Monsieur René Samuel Sirat grand rabbin de France. Il a été réalisé à l'initiative du conseil d'administration de la communauté juive de Lille présidé par Monsieur Jacques Malamet $»$.

${ }^{47}$ AML : 1 P 9.
} 
du mobilier et des lustres en 2013, les mesures prises à partir des années 2000 pour renforcer la sécurité aux abords du lieu et faire face à la menace terroriste, l'institution de cycles de conférences ouvertes au public ou encore la nouvelle campagne, pour réaliser d'amples travaux, menée entre 2018 et $2020^{48}$ et qui a permis de rénover entièrement la synagogue en mettant à jour les décorations primitives - l'inauguration était prévue en novembre 2020 mais finalement fut reportée en raison des mesures prises contre l'épidémie de covid-19 - et la diversification des activités et des usages de la synagogue attestent des multiples manières d'être juif à Lille et dans sa région aujourd'hui.

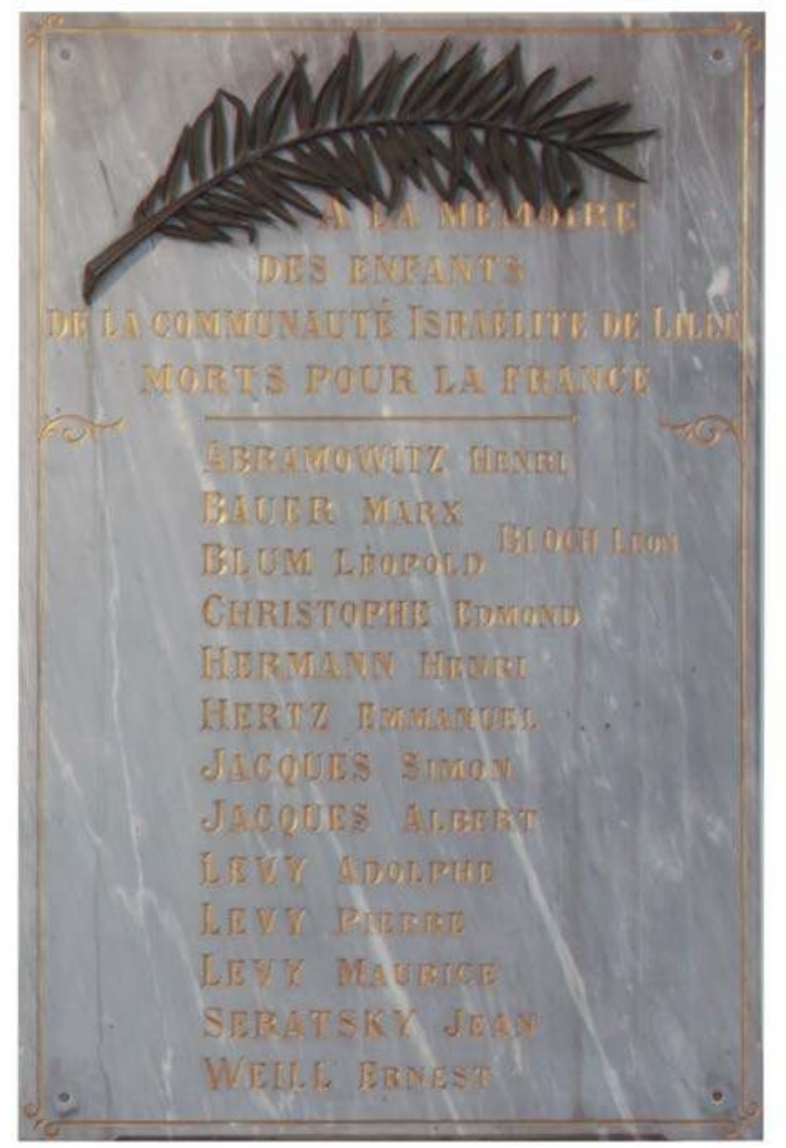

Plaque en l'honneur des Juifs morts à la Première Guerre mondiale. Photo StudioptiK ${ }^{49}$

\footnotetext{
${ }^{48}$ Lille Actu, 26 novembre 2018.

${ }^{49}$ Remerciements à l'agence de communication et de création graphique StudioptiK (Marquette-lez-Lille) ainsi qu'à Messieurs Guy Bensoussan et Élie Dahan, pour nous avoir permis d'utiliser leurs photos.
} 


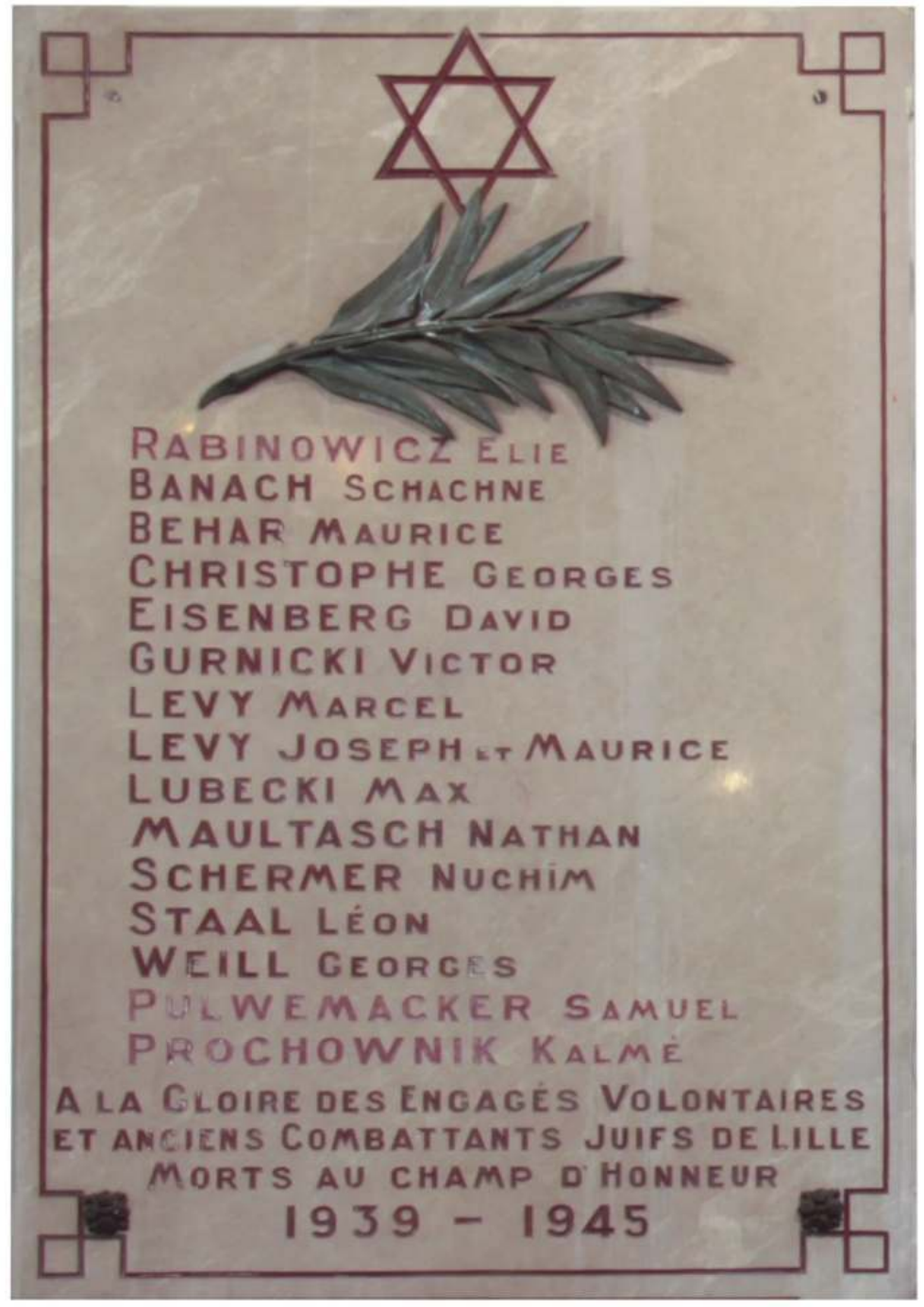

Plaque en l'honneur des Juifs décédés lors des combats de la Seconde Guerre mondiale. Photo StudioptiK 


\begin{tabular}{|c|c|c|}
\hline 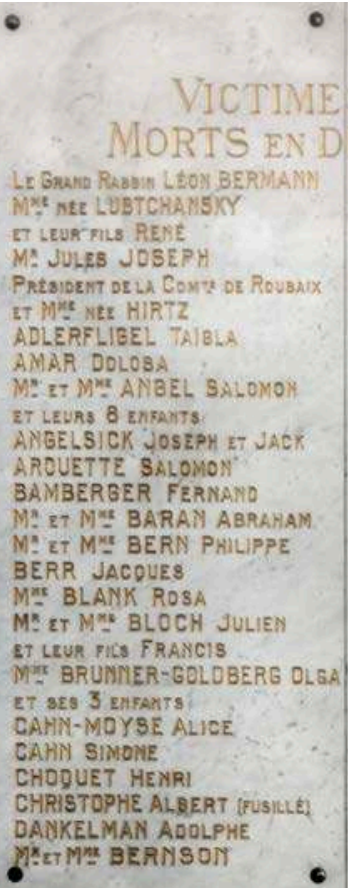 & 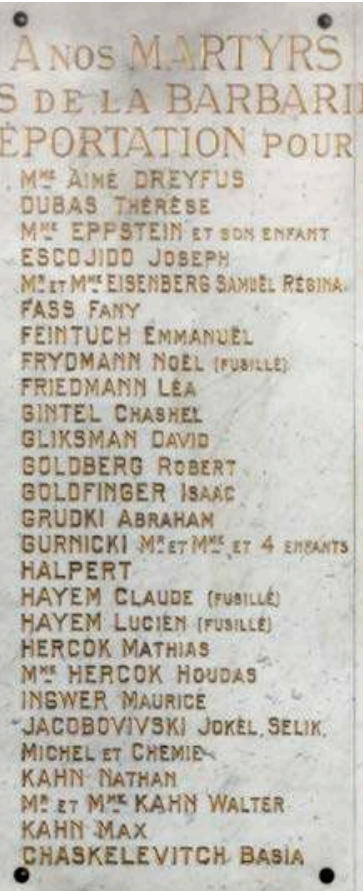 & 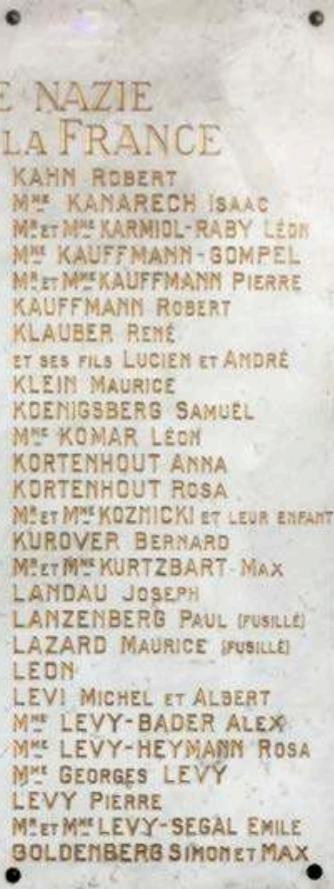 \\
\hline
\end{tabular}

\begin{tabular}{|c|c|c|}
\hline 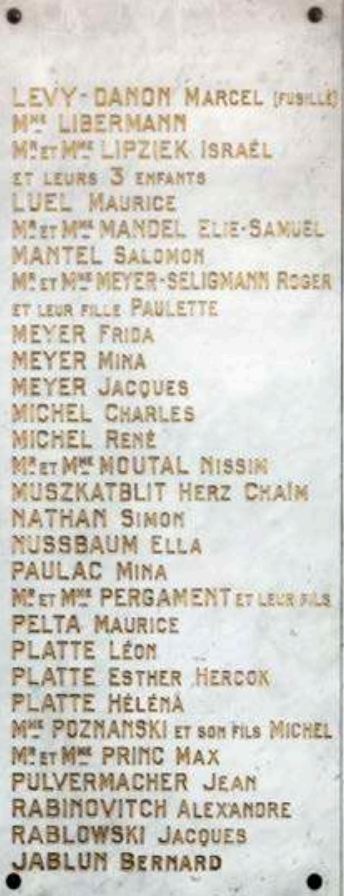 & 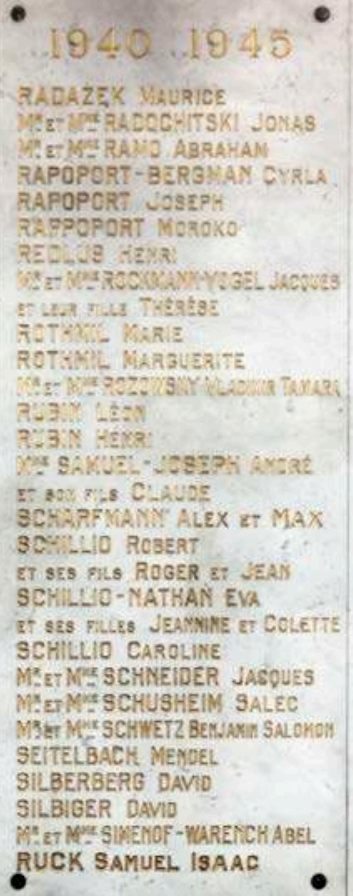 & 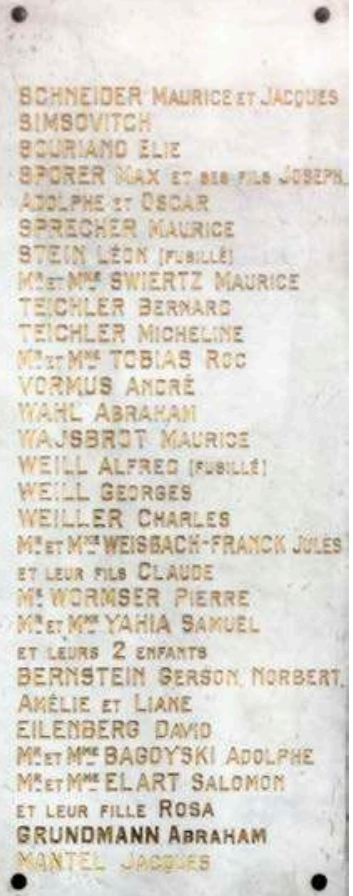 \\
\hline
\end{tabular}

Plaques commémoratives de la déportation des Juifs de Lille.

Elles sont disposées de part et d'autre d'une porte latérale de la synagogue.

Remarque : la mention « fusillé » au nom des Marcel Levy-Danon.

Photo StudioptiK 


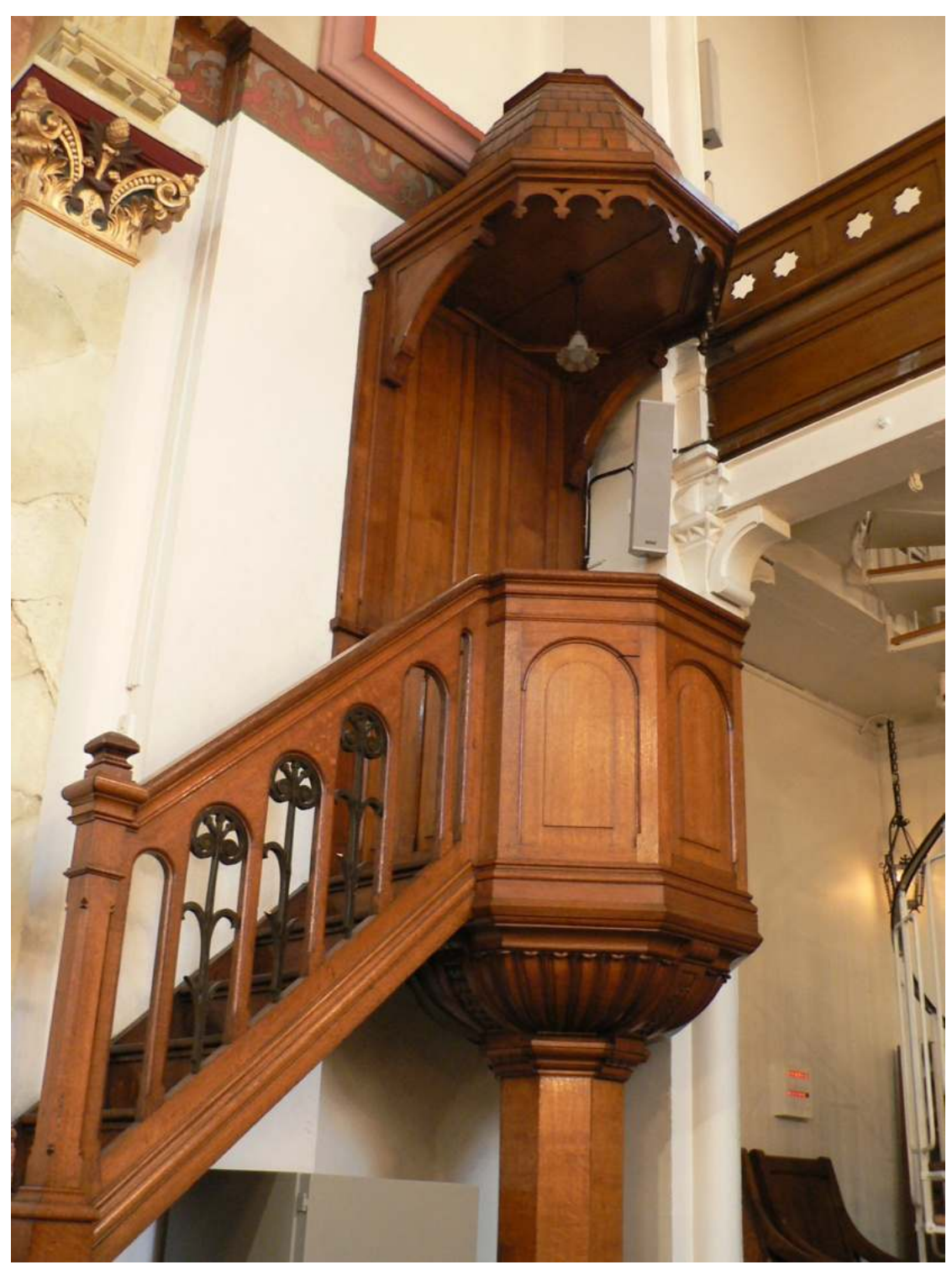

Chaire du rabbin avant la rénovation de 2020.

Un enduit blanc masquait les peintures murales primitives.

La chaire n'est plus utilisée depuis des décennies.

Photo Élie Dahan 
Tsafon 81 : La synagogue de Lille et les deux guerres mondiales

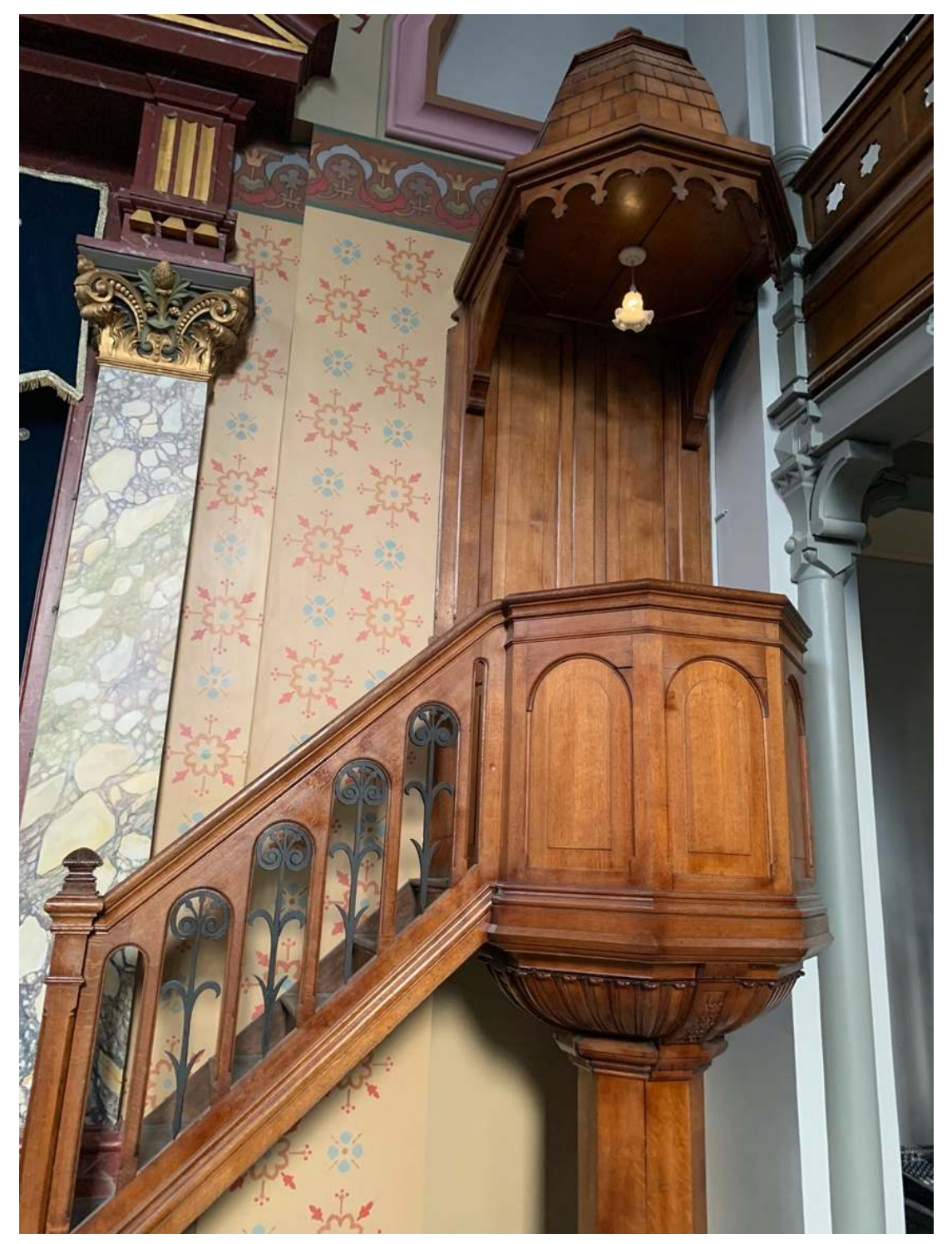

Chaire du rabbin après la rénovation de 2020 .

Non seulement l'escalier a été rénové (ferronnerie) mais les peintures murales primitives ont été restaurées. Photo Danielle Delmaire 


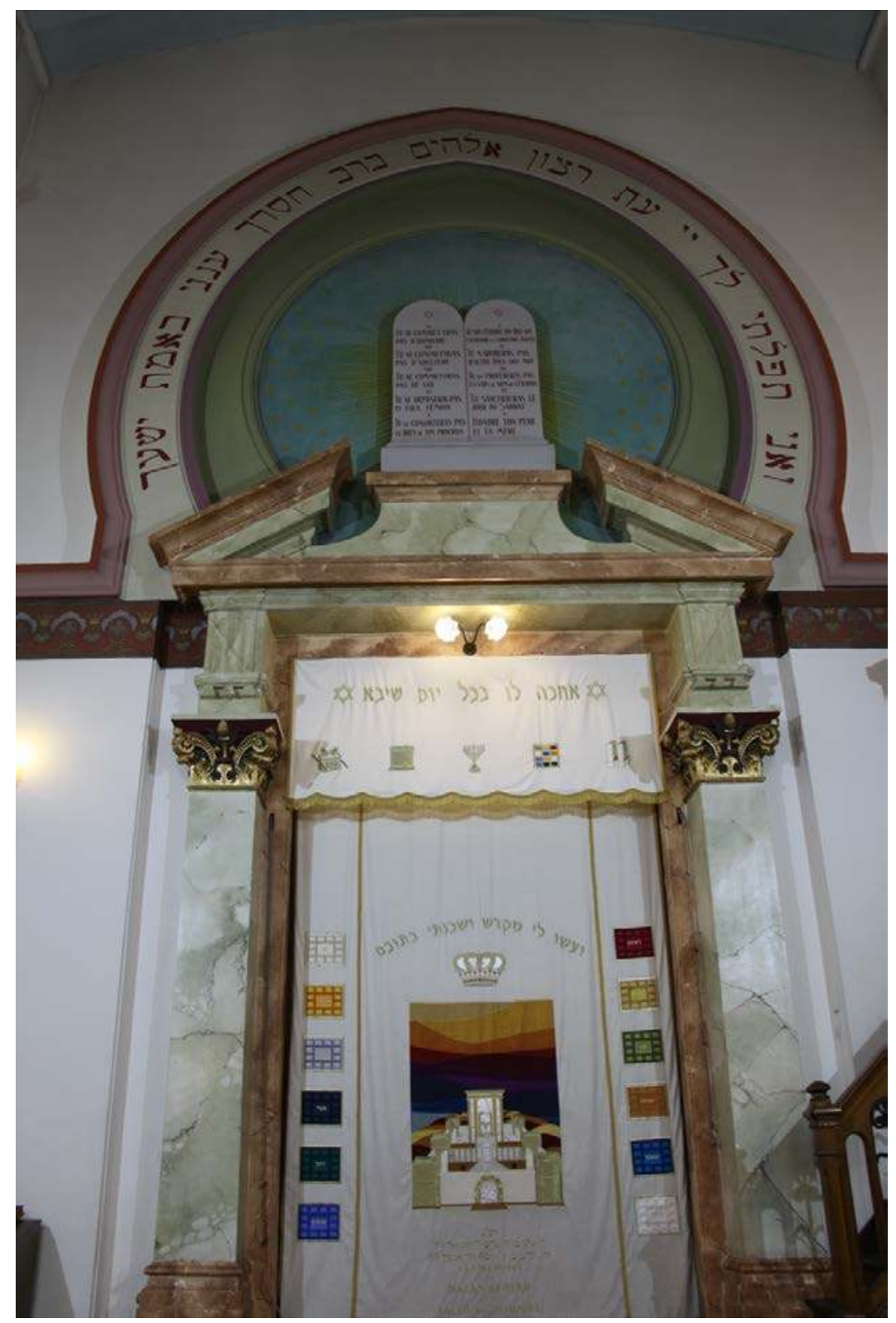

L'arche sainte ou aron haqodesh avant la rénovation de 2020. Le même enduit blanc masquait les peintures murales primitives. Photo Élie Dahan 
Tsafon 81 : La synagogue de Lille et les deux guerres mondiales

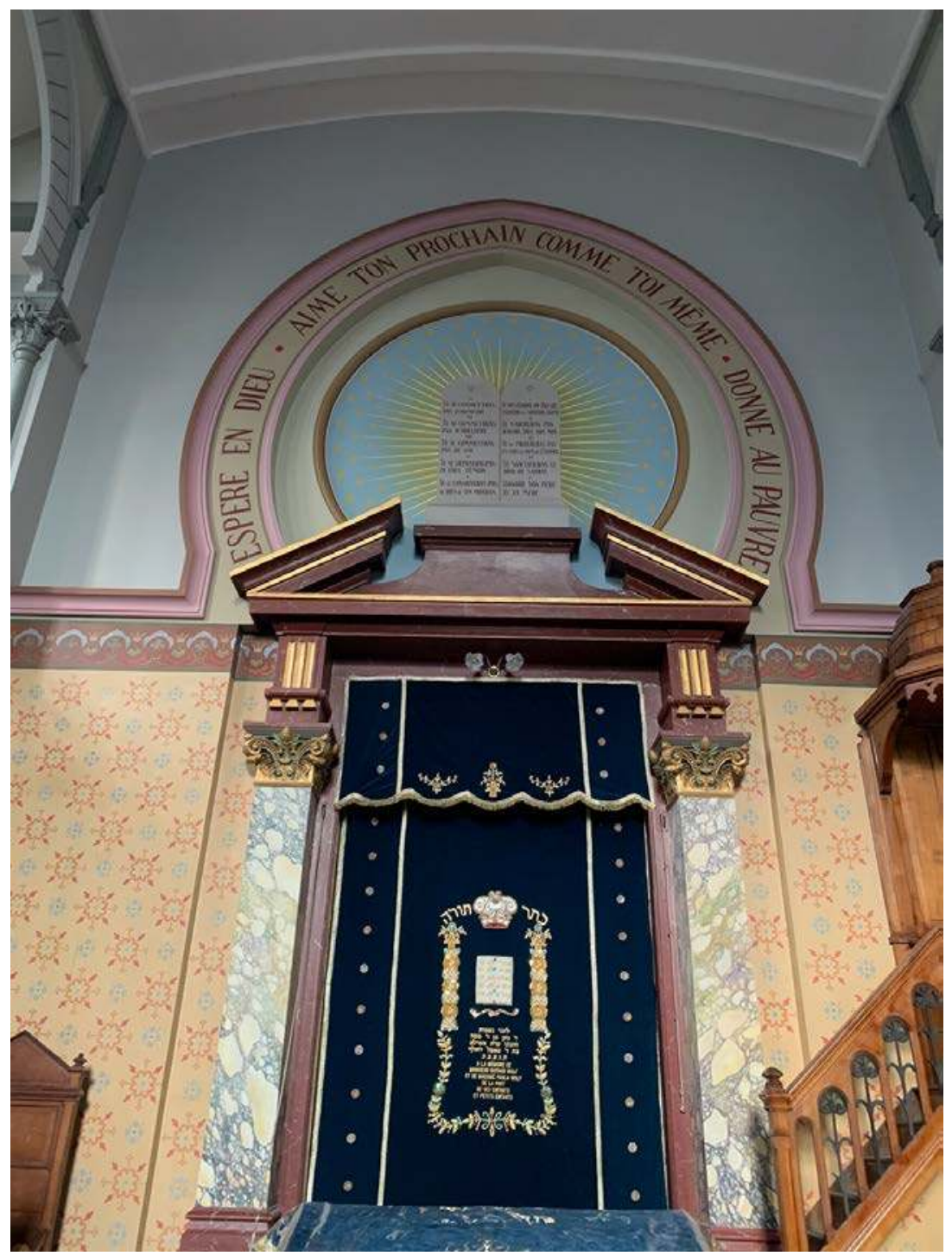

L'arche sainte après la rénovation de 2020.

Le texte biblique au-dessus de l'arche a été réécrit en français comme il l'avait été originellement.

Les peintures murales primitives sont restaurées.

Photo Danielle Delmaire 